\title{
Thermocapillary migration and interaction of two nondeformable drops*
}

\author{
Zhao-hua YIN (尹兆华), Lei CHANG (常 否), \\ Wen-rui HU (胡文瑞), Peng GAO (高 鹏) \\ (National Microgravity Laboratory, Institute of Mechanics, Chinese Academy of Sciences, \\ Beijing 100190, P. R. China) \\ (Contributed by Wen-rui HU)
}

\begin{abstract}
A numerical study on the interaction of two spherical drops in the thermocapillary migration is presented in the microgravity environment. Finite-difference methods are adopted. The interfaces of the drops are captured by the front-tracking technique. It is found that the arrangement of the drops directly influences their migration and interaction, and the motion of one drop is mainly determined by the disturbed temperature field because of the existence of the other drop.
\end{abstract}

Key words interaction of two drops, thermocapillary motion, front tracking method

Chinese Library Classification $\quad \mathrm{O}_{3} 59^{+} .1$

2010 Mathematics Subject Classification 76T99, 76M20

\section{Introduction}

Under the microgravity condition, the thermocapillary migration of drops or bubbles in the matrix liquid is caused by the nonuniform interface tension introduced by the temperature gradient. The motion is of great importance in material processing and other applications in space. The original work in this field was performed by Young et al. ${ }^{[1]}$. In their study, the inertial convection and the thermal convection were neglected (i.e., the so-called YoungGoldstein-Block (YGB) model). The derived migration velocity is

$$
V_{\mathrm{YGB}}=\frac{2 U}{\left(2+\frac{3 \mu_{\mathrm{d}}}{\mu_{\mathrm{b}}}\right)\left(2+\frac{k_{\mathrm{d}}}{k_{\mathrm{b}}}\right)} .
$$

Here, $k$ is the thermal conductivity, and $U$ is the reference velocity defined by the balance of the thermocapillary force and the viscosity force on the drop/bubble as follows:

$$
U=\left|\sigma_{T}\right|\left|\nabla T_{\infty}\right| \frac{a}{\mu_{\mathrm{b}}}
$$

* Received Apr. 14, 2011 / Revised May 4, 2011

Project supported by the Knowledge Innovation Program of the Chinese Academy of Sciences (No. KJCX2-YW-L08)

Corresponding author Zhao-hua YIN, Associate Professor, Ph. D., E-mail: zhaohua.yin@imech.ac.cn 
where $\mu_{\mathrm{b}}$ is the kinematic viscosity, $\sigma_{T}$ is the rate of the change of the interfacial tension with temperature, $\nabla T_{\infty}$ is the temperature gradient imposed on the matrix liquid, and $a$ is the radius of the drop/bubble. The symbols with the subscript $d$ mean the parameters of the drop/bubble, and the parameters of the bulk liquid are indicated by the subscript b.

There are many other studies on the thermocapillary motion of isolated drops/bubbles (see [2] and the references therein). In practice, it is common to have two or more drops/bubbles in the continuous phase. Therefore, it is necessary to study their interaction. The first axisymmetric investigation of two thermocapillary bubbles was conducted by Meyyappan et al. ${ }^{[3]}$ using the bipolar coordinates. It was found that the smaller bubble always moved faster than the isolated drop while the bigger one moved slightly slower. Meyyappan and Subramanian ${ }^{[4]}$ extended the above work to the arbitrarily placed bubbles. Balasubramaniam and Subramanian ${ }^{[5]}$ assumed that two bubbles migrated in the potential flow (namely, the related Reynolds number $(R e)$ is very large). Moreover, the matched asymptotic analysis was adopted to solve the energy equation with large Marangoni numbers $(M a)$. It was found that the thermal wake of the leading bubble would disturb the temperature field around the trailing bubble and reduce its velocity. The interaction between two spherical drops was first studied by Anderson with a reflection method ${ }^{[6]}$. It was found that the interaction between the drops driven by the thermocapillary effects was much weaker than that of the sedimentation. Keh and Chen ${ }^{[7]}$ analyzed the axisymmetric motion of two drops in the bishperical coordinates. Their later combined analytical-numerical study ${ }^{[8]}$ was about a finite chain of spherical drops along the line of their centers. The interaction of two deformable drops in the axisymmetric coordinates was studied by Zhou and Davis ${ }^{[9]}$. The thermocapillary interaction of drops or bubbles toward a hot wall at the finite $R e$ and $M a$ was numerically studied by Nas and Tryggvason ${ }^{[10]}$ and Nas et al. ${ }^{[11]}$. It was found that the bubbles and light drops lined up perpendicular to the temperature gradient and were evenly spaced in the horizontal direction. A space experiment showed that a small leading drop could retard the movement of the big trailing drop ${ }^{[12]}$.

So far, there is no systematic study on the interaction of two arbitrarily placed drops in the thermocapillary migration. It is the main subject in this paper. We focus our investigation on two drops with the same size and the same physical parameters (kinematic viscosity, thermal diffusivity, density, and specific heat). The governing equations and numerical methods are introduced in Section 2, the detailed numerical models and parameters of the simulations are presented in Section 3, and the results with and without the inertia and thermal convection are discussed in Sections 4 and 5, respectively. The conchusions are presented in Section 6.

\section{Governing equations and numerical methods}

It can be seen from Fig. 1 that, in the thermocapillary motion, the two drops with the same radius $a$ are surrounded by the bulk fluid in a rectangular box $\Omega=\left[x_{0}, x_{1}\right] \times\left[y_{0}, y_{1}\right] \times\left[z_{0}, z_{1}\right]$. The box is closed by the no-slip walls. The direction of the temperature gradient is along the $z$-axis, and $x=0$ is treated in the drop centers. The governing equations for this problem are

$$
\begin{aligned}
& \nabla \cdot \boldsymbol{u}=0 \\
& \frac{\partial(\rho \boldsymbol{u})}{\partial t}+\nabla \cdot(\rho \boldsymbol{u u})=-\nabla p+\nabla \cdot\left(\mu\left(\nabla \boldsymbol{u}+\nabla^{\mathrm{T}} \boldsymbol{u}\right)\right)+\boldsymbol{F}_{\sigma} \\
& \rho C_{p}\left(\frac{\partial T}{\partial t}+\boldsymbol{u} \cdot \nabla T\right)=\nabla \cdot(k \nabla T) .
\end{aligned}
$$

Here, $\boldsymbol{u}=(u, v, w), \boldsymbol{x}=(x, y, z) \in \Omega$, and $\boldsymbol{F}_{\sigma}$ is the body force term calculated by integrating the surface tension across the interface ${ }^{[2]}$. The above conservative equations are valid for both the drop phase and the bulk phase with the different material parameters for the different 


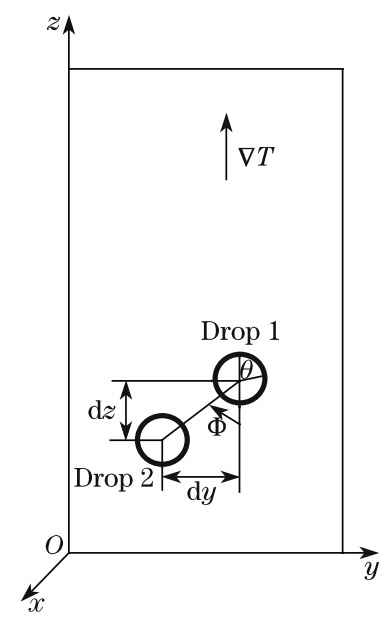

Fig. 1 Sketch of two drops in the thermocapillary migration ( $\Phi$ is the angle between the temperature gradient and the center line of two drops)

phases. We define the nondimensional quantities as

$$
\left\{\begin{array}{l}
\overline{\boldsymbol{u}}=\frac{\boldsymbol{u}}{U}, \quad \overline{\boldsymbol{x}}=\frac{\boldsymbol{x}}{a}, \quad \bar{t}=\frac{t}{\frac{a}{U}}, \quad \bar{p}=\frac{p}{\rho_{\mathrm{b}} U^{2}}, \\
\bar{T}=\frac{T}{\left|\nabla T_{\infty}\right| a}, \quad \bar{\rho}=\frac{\rho}{\rho_{\mathrm{b}}}, \quad \bar{\mu}=\frac{\mu}{\mu_{\mathrm{b}}}, \quad \bar{k}=\frac{k}{k_{\mathrm{b}}}, \\
\bar{C}_{p}=\frac{C_{p}}{C_{p \mathrm{~b}}}, \quad \overline{\boldsymbol{F}}_{\sigma}=\frac{\boldsymbol{F}_{\sigma} a}{\rho_{\mathrm{b}} U^{2}}, \quad R e=\frac{U a}{\nu_{\mathrm{b}}}, \quad M a=\frac{U a}{\kappa_{\mathrm{b}}} .
\end{array}\right.
$$

Here, $\nu_{\mathrm{b}}=\mu_{\mathrm{b}} / \rho_{\mathrm{b}}$ is the kinematic viscosity, and $\kappa_{\mathrm{b}}=k_{\mathrm{b}} /\left(\rho_{\mathrm{b}} C_{p \mathrm{~b}}\right)$ is the thermal diffusivity of the matrix liquid. The nondimensional equations can be written as

$$
\begin{aligned}
& \nabla \cdot \overline{\boldsymbol{u}}=0, \\
& \frac{\partial(\bar{\rho} \overline{\boldsymbol{u}})}{\partial \bar{t}}+\nabla \cdot(\bar{\rho} \overline{\boldsymbol{u}} \overline{\boldsymbol{u}})=-\nabla \bar{p}+\frac{1}{R e} \nabla \cdot\left(\bar{\mu}\left(\nabla \overline{\boldsymbol{u}}+\nabla^{\mathrm{T}} \overline{\boldsymbol{u}}\right)\right)+\overline{\boldsymbol{F}}_{\sigma}, \\
& \bar{\rho} \bar{C}_{p}\left(\frac{\partial \bar{T}}{\partial \bar{t}}+\overline{\boldsymbol{u}} \cdot \nabla \bar{T}\right)=\frac{1}{M a} \nabla \cdot(\bar{k} \nabla \bar{T}) .
\end{aligned}
$$

The boundary conditions for the velocities are

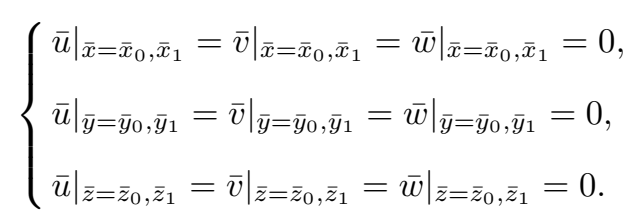

For the energy equation, the Dirichlet boundary conditions are adopted as follows:

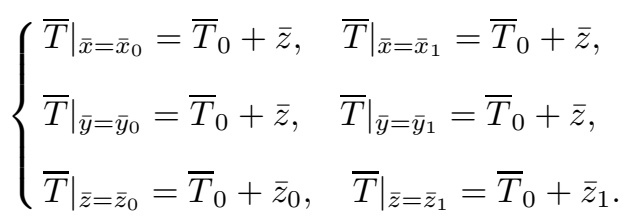


The initial conditions are

$$
\left\{\begin{array}{l}
\left.\bar{u}\right|_{\bar{t}=0}=\left.\bar{v}\right|_{\bar{t}=0}=\left.\bar{w}\right|_{\bar{t}=0}=0 \\
\left.\bar{T}\right|_{\bar{t}=0}=\bar{T}_{0}+\bar{z}
\end{array}\right.
$$

In the following, the symbols without overbars are adopted to denote the nondimensional values.

\section{$3 \quad$ Numerical models and parameters}

Figure 1 indicates the initial positions of the two drops. The horizontal and vertical distances between the two drops are $\mathrm{d} y$ and $\mathrm{d} z$, respectively. The traditional definitions of the nondimensional distances are defined as $S_{y}=\mathrm{d} y / 2$ and $S_{z}=\mathrm{d} z / 2^{[7]}$, and $S_{y 0}$ and $S_{z 0}$ denote the initial values of $S_{y}$ and $S_{z}$, respectively. $\theta$ indicates the point on the drop interface in the $x=0$ plane, $\theta=0$ is the front stagnation, and $\theta=\pi$ or $\theta=-\pi$ is the rear stagnation. The points in the clockwise direction from the front stagnation are denoted with $\theta>0$. Otherwise, $\theta<0$. In the full three-dimensional model, the computation zone is set to be $6 \times 9 \times 24$ on the grid of $60 \times 90 \times 240$. The time steps are $10^{-6}$ for $R e=M a=10^{-3}$ and $10^{-3}$ for other $R e$ and $M a$ values. To save the computing time, the axisymmetric model is adopted in the case of $\Phi=0$. It has been realized that some nonaxisymmetrical behaviors might arise for the isolated drop when the full three-dimensional simulating domain is fairly small ${ }^{[13]}$. In this paper, we adopt a fairly large domain, and we assume that the nonaxisymmetrical effect can be neglected for moderate parameters. In the axisymmetric simulations, drop 1 in the hotter region is called the leading drop, drop 2 in the colder region is called the trailing drop, and $S$ and $S_{0}$ are adopted to replace $S_{z}$ and $S_{z 0}$ in the three-dimensional model, respectively. The computing domain is $6 \times 24$ with the resolution of $128 \times 512$. The time steps are $5 \times 10^{-7}$ for the simulation of $R e=M a=10^{-3}$ and $2 \times 10^{-4}$ for other $R e$ and $M a$ values, respectively. In this paper, all material parameters of the two drops are assumed to be the same.

Generally speaking, the nondimensional thermocapillary migration velocities of the drops are quite small (about 0.1), and the usually defined nondimensional velocity (namely, $\overline{\boldsymbol{u}}=\boldsymbol{u} / U_{\max }$, where $U_{\max }$ is the maximum velocity in the flow field instead of $\left.U=\left|\sigma_{T}\right|\left|\nabla T_{\infty}\right| a / \mu_{\mathrm{b}}\right)$ is even lower. The influence of $R e$ in the present study is trivial and can be inferred from the results of the isolated drop. The role of $R e$ will not be discussed here. We simply set that $R e=1$ if not specified, and we will concentrate on the influence of the thermal convection and the initial distance.

To have a clear idea of the interaction of two thermocapillary drops, it is necessary to study the isolated drop. In the following, the velocity of the isolated drop is denoted as $W_{\text {iso }}$.

\section{Thermocapillary migration of two drops without inertia and thermal convection}

In this section, the small $R e$ and $M a\left(10^{-3}\right)$ are adopted in the simulation in comparison with some previous analytical results. Figure 2 shows the final migration velocities for $\Phi=0$, $\pi / 2$. In the case of $\Phi=0$, the velocities of the two drops are very close but faster than $W_{\text {iso }}$ (see the solid line in Fig. 2). This phenomenon was also found in the previous analytical and numerical studies ${ }^{[7,14]}$. In the case of $\Phi=\pi / 2$, our three-dimensional simulations show that the drop velocities in the $y$ - or $x$-direction are neglectable. The $z$-direction velocities $(W)$ of both drops are still the same, but they are slower than $W_{\text {iso }}$ (see the dash line in Fig. 2). In both cases, the increase of the initial drop distance makes the final drop velocity closer to $W_{\text {iso }}$.

Similarly, there are lots of studies on the interaction between two rigid spheres ${ }^{[15-17]}$. Using the previous analytical linear results, we compare the difference between the drops and the rigid spheres. Assume that the rigid sphere/drop has a constant velocity $U$, and the original point is 


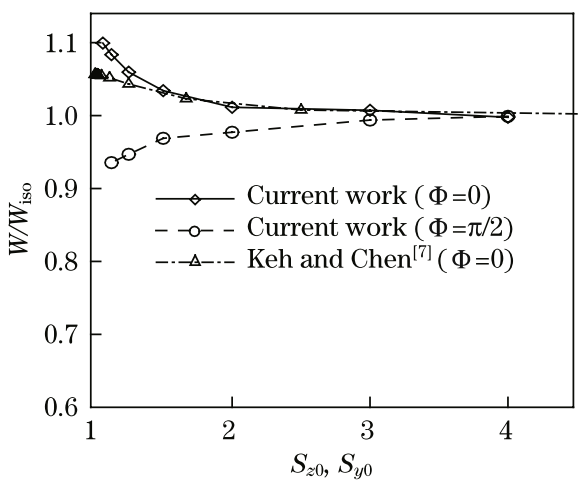

Fig. 2 Steady-state thermocapillary migration velocities of two drops with different initial distances (the horizontal coordinates are $S_{z 0}$ for $\Phi=0$ and $S_{y 0}$ for $\Phi=\pi / 2$, and $R e=M a=10^{-3}$ )

in the center of the rigid sphere/drop. In the spherical coordinates $(r, \theta, \phi)$, the potential flow describes the thermocapillary motion of the drop written as ${ }^{[18-20]}$

$$
v_{r}=U \frac{a^{3}}{r^{3}} \cos \theta, \quad v_{\theta}=-\frac{1}{2} U \frac{a^{3}}{r^{3}} \sin \theta .
$$

Here, $\theta=0$ is the motion direction. The motion of the rigid sphere is described by the Stokes flow ${ }^{[20-21]}$ as follows:

$$
v_{r}(r, \theta)=\frac{1}{2} U\left(3 \frac{a}{r}-\frac{a^{3}}{r^{3}}\right) \cos \theta, \quad v_{\theta}(r, \theta)=-\frac{1}{4} U\left(3 \frac{a}{r}+\frac{a^{3}}{r^{3}}\right) \sin \theta .
$$

It is clear that, with the increase of $r$, the velocities for the rigid sphere and the drop decay in the magnitudes of $O\left(1 / r^{3}\right)$ and $O(1 / r)$, respectively. The velocity perturbation in the potential flow spreads in a relatively small region (see Fig. 3(a)) and has the obvious directionality, because the liquid in the front of the moving drop will be supplied to the back of the drop. For the Stokes flow, the velocity perturbation spreads in a fairly large region, and the surrounding liquid tries to move with the sphere (see Fig. 3(b)).

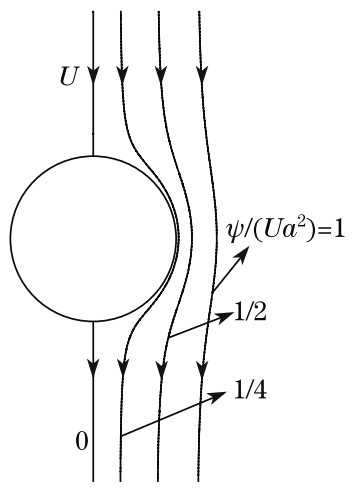

(a) Potential flow

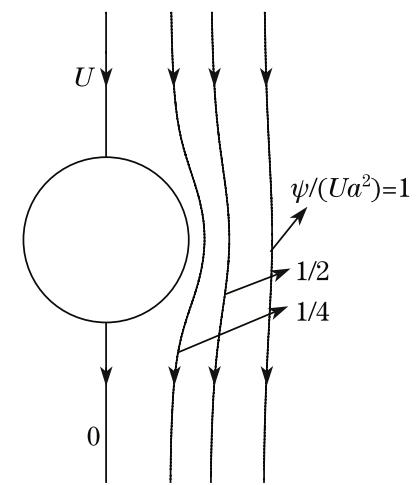

(b) Stokes flow

Fig. 3 Streamlines in a reference frame attached to the drop/sphere

The drag coefficients of both rigid spheres are always lower than that of the isolated sphere ${ }^{[22-23]}$, which means that two spheres move faster than one isolated sphere. When the 
nonlinear effect is strong, the main interest in the interaction between rigid spheres is the drag coefficients changed by the wake flow behind the leading body. However, in the thermocapillary study, the disturbed temperature field is the most important, and we will concentrate on it in the following section.

\section{Thermocapillary migration of two drops with finite inertia and thermal convection}

\subsection{Influence of thermal convection for $\boldsymbol{\Phi}=\mathbf{0}$}

First, we study the influence of $M a$ on the interaction between two drops in the thermocapillary motion with $S_{0}=1.5$ and $R e=1$.

In the case of $M a=1$, the leading drop is faster than the trailing drop, but both of them move faster than the isolated drop (see Fig. 4(a)). This is similar to that we have discussed in the last section. For $M a=20$, the leading drop is faster than the isolated drop throughout the simulation, while the velocity of the trailing drop is up to $8 \%$ lower than $W_{\text {iso }}$ (see Fig. $4(\mathrm{~b})$ ). For $M a=100$, the trailing drop is even slower, and its velocity is up to $20 \%$ lower than $W_{\text {iso }}$ (see Fig. 4(c)).

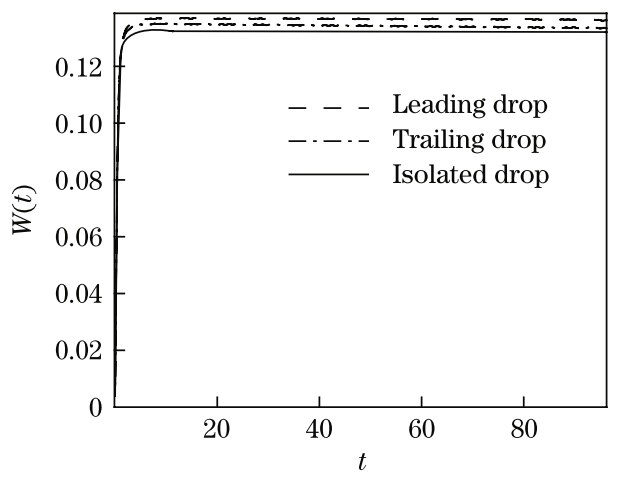

(a) $M a=1$

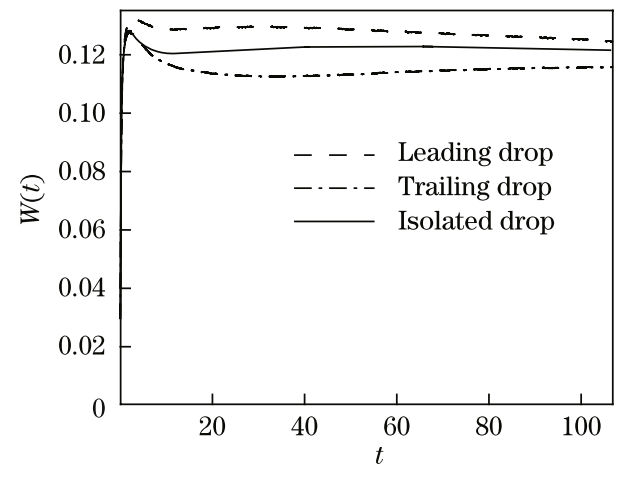

(b) $M a=20$

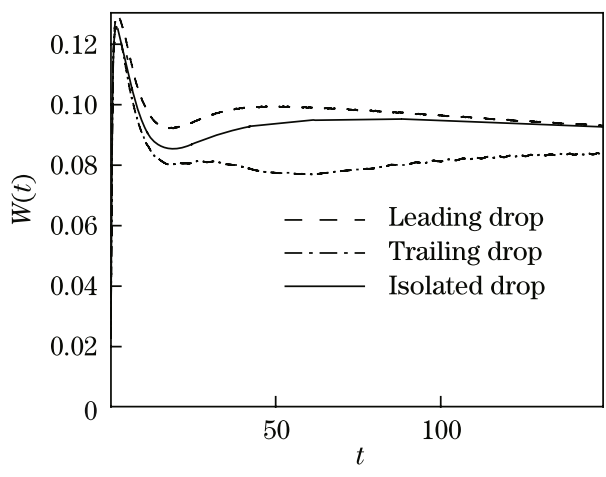

(c) $M a=100$

Fig. 4 Migration velocities of the leading drop, the trailing drop, and the isolated drop with $R e=1$ and $S_{0}=1.5$

Figure 5 shows the isotherms around the drops. When $M a$ is small, the isotherms around the drops are almost straight and evenly spaced throughout the simulation (see Figs. 5(a)-5(d)). When $M a$ is increased, the isotherms near $r=0$ arch to the hotter region, and there is a closed cold zone arising in the drop (see Figs. $5(\mathrm{c})-5(\mathrm{f})$ ). The temperature at the rear stagnation point of the leading drop is lower than that of the isolated drop, and the temperature gradient of the 
trailing drop is also reduced.

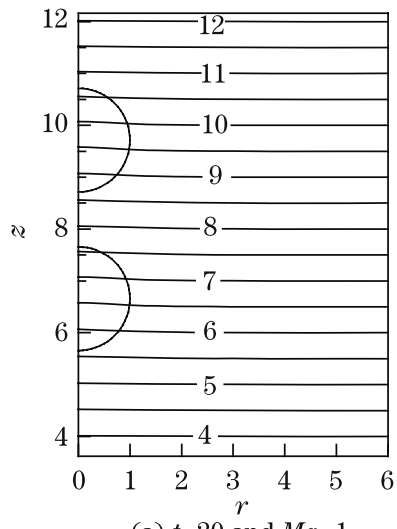

(a) $t=20$ and $M a=1$

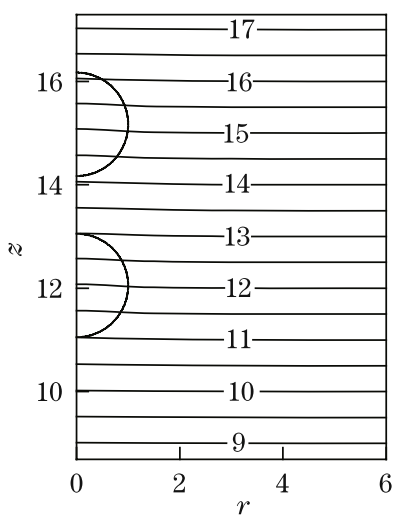

(d) $t=60$ and $M a=1$

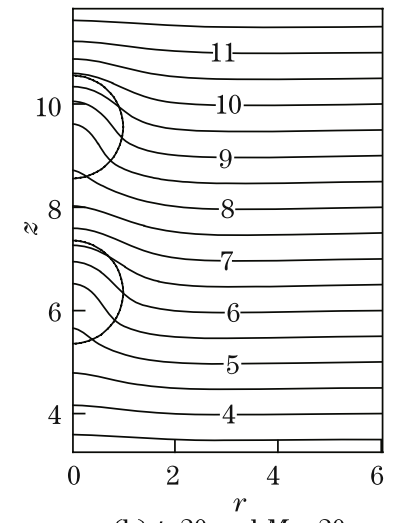

(b) $t=20$ and $M a=20$

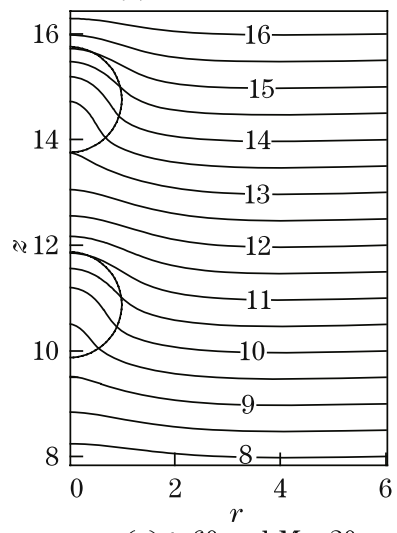

(e) $t=60$ and $M a=20$

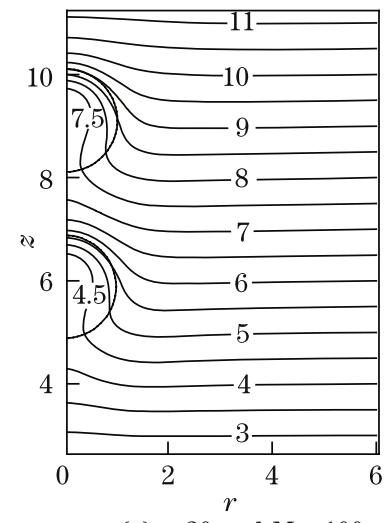

(c) $t=20$ and $M a=100$

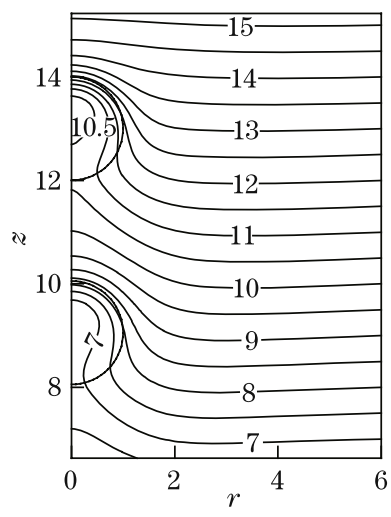

(f) $t=60$ and $M a=100$

Fig. 5 Isotherms around the two drops with $R e=1$ and $S_{0}=1.5$

Figure 6 shows the temperature difference between the point on the drop surface and the front stagnation (to get a clear idea of this difference, the corresponding value of the isolated drop is subtracted). It can be seen that the temperature difference between the front and rear stagnation points of the leading drop (solid line) is larger than that of the isolated drop. On the contrary, the difference for the trailing drop (dashed line) is smaller than that of the isolated drop and decreases with the increasing $M a$. Figure 6 clearly shows that the influence of the thermal convection on the trailing drops is much stronger than that on the leading drops.

When the heat convection is stronger, the influence on the trailing drop is also bigger. Hence, the separated distances $\left(S-S_{0}\right)$ increase more rapidly for the larger $M a$ (see Fig. 7 ). Note that the separating velocity of $M a=100$ before $t=60$ is lower than that of $M a=20$. This is because the fluctuation process of the migrating velocity in the beginning is longer for the larger $M a^{[2]}$. For example, the thermal wake left by the leading drop with $M a=100$ is not fully developed until $t=60$ (see Figs. $5(\mathrm{c})$ and $5(\mathrm{f})$ ), and the velocity difference between the leading and trailing drops is not so large.

The temperature differences for $M a=20$ at various moments are shown in Fig. 8 . At $t=20$, the temperature difference of the leading drop becomes bigger than that of the isolated drop and starts to become smaller afterwards. When the thermal convection effect and the temperature disturbance caused by the leading drop are fully developed, the temperature difference of the trailing drop reaches its minimum at $t=40$ and starts to become larger afterwards. 


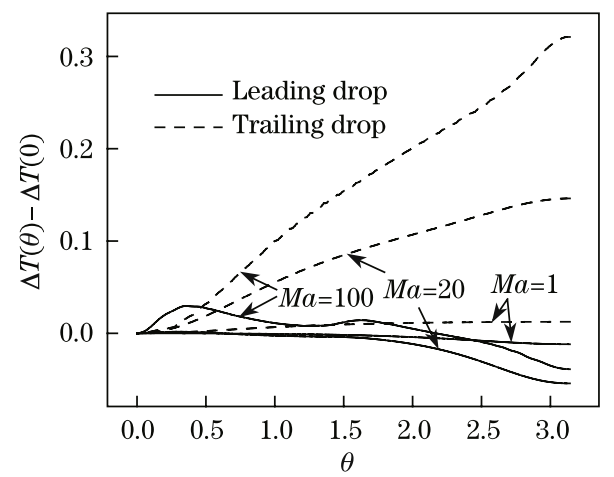

Fig. 6 Temperature difference between the point $\theta$ on the interface and the front stagnation at $t=60$ for $R e=1, S_{0}=$ 1.5, and $\Delta T(\theta)=T(\theta)-T_{\text {iso }}(\theta)$

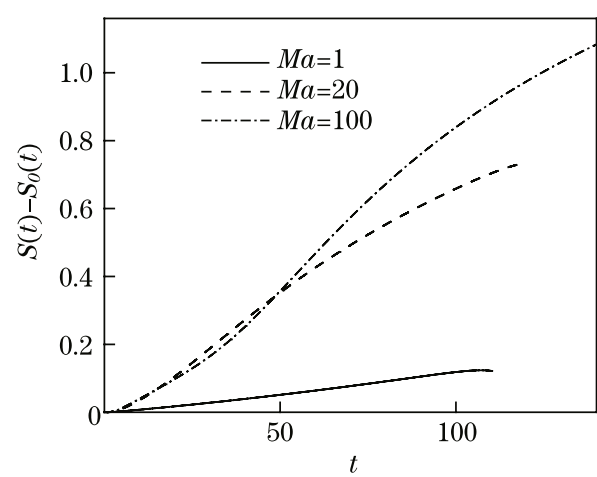

Fig. 7 Time evolutions of separation distances between two drops for $R e=1$ and $S_{0}=1.5$

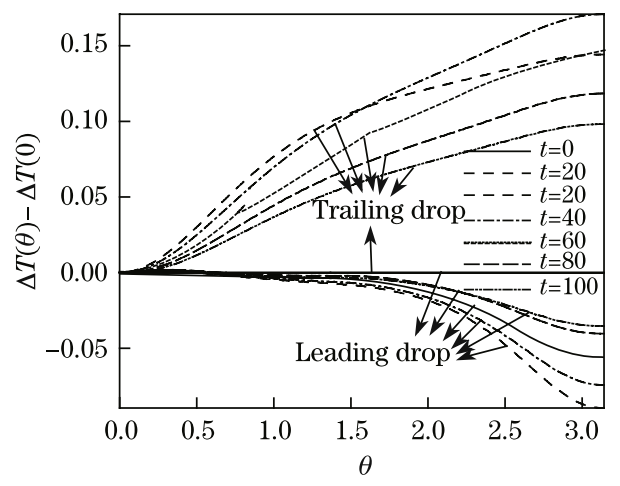

Fig. 8 Time evolution of the temperature difference for $R e=1, M a=20$, and $S_{0}=1.5$

\subsection{Influence of initial distance for $\Phi=0$}

Obviously, there will be no interaction between the drops if they are far enough from each other. Therefore, it is interesting to know the critical initial distance, at which both drops migrate like an isolated one.

Several different initial distances are tested for the case of $R e=1$ and $M a=20$ (see Fig. 9). The curve of the leading drop with $S_{0}=3$ is almost identical to that of the isolated drop (see Fig. 9(a)), while the critical initial distance is 5 for the trailing drop (see Fig. 9(b)). It seems that the thermal wake left by the leading drop affects the trailing drop at a distance that is longer than the one at which the trailing drop interferes the leading drop.

When $M a$ is increased to 100 , the critical initial distance for the leading drop remains to be $S_{0}=3$ (see Fig. 10(a)). Moreover, the thermal wake left by the leading drop is much longer, and the critical initial distance for the trailing drop is longer than 5 .

Figure 11 shows the time evolutions of the distances between the two drops. It is clear that the smaller the initial distance between the two drops is, the faster they will separate from each other. In the later stage of the simulations, it seems that the distance differences caused by various initial distances vanish, and the final distance between the drops (of course, if they are not too far away apart) is determined by other parameters. 


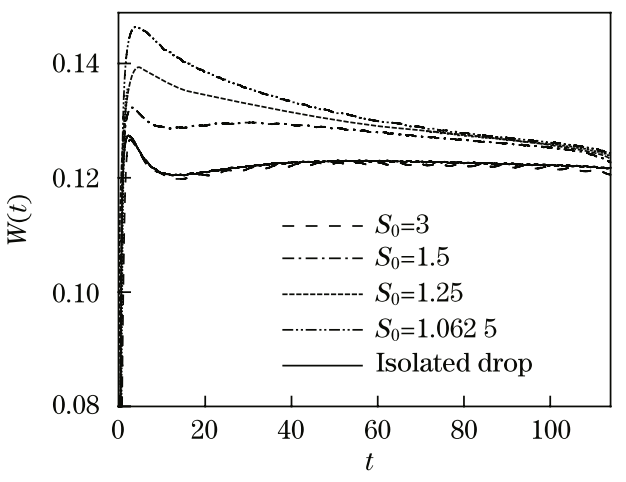

(a) Leading drop

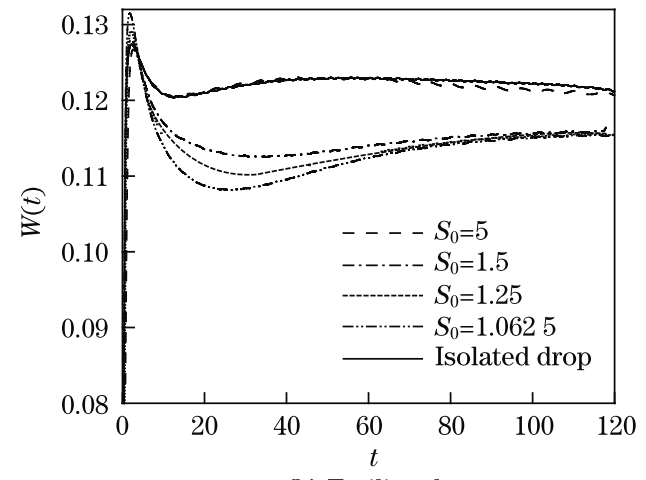

(b) Trailing drop

Fig. 9 Time evolutions of the separating distances between the drops

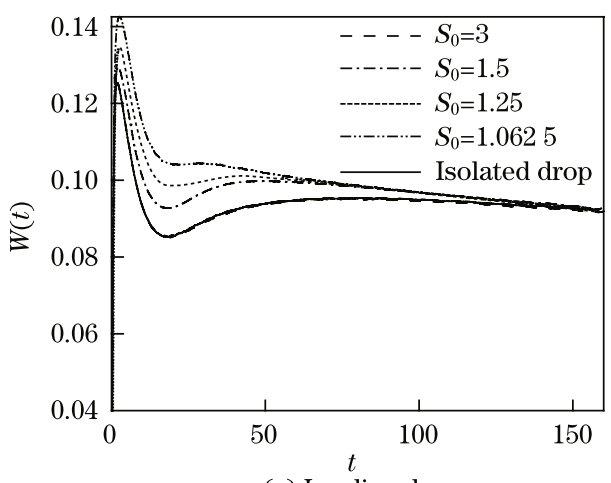

(a) Leading drop

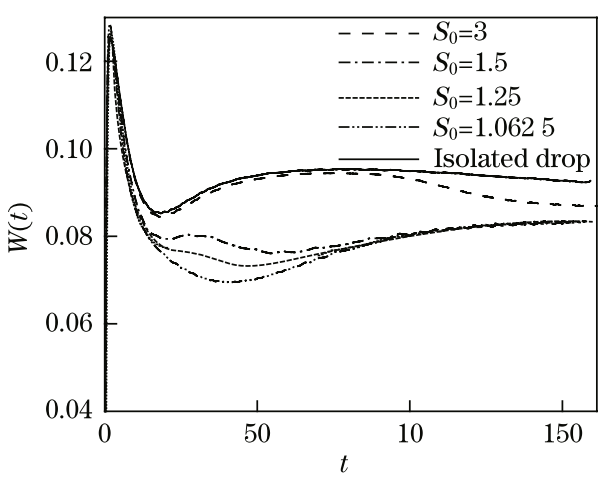

(b) Trailing drop

Fig. $10 R e=1$ and $M a=100$ time evolutions of drop velocities with various initial distances

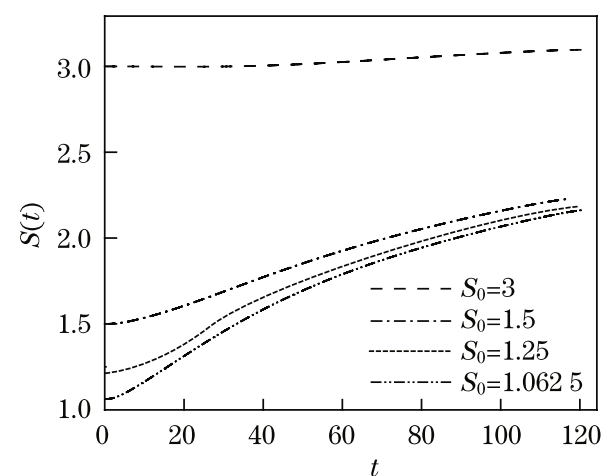

(a) $R e=1$ and $M a=20$

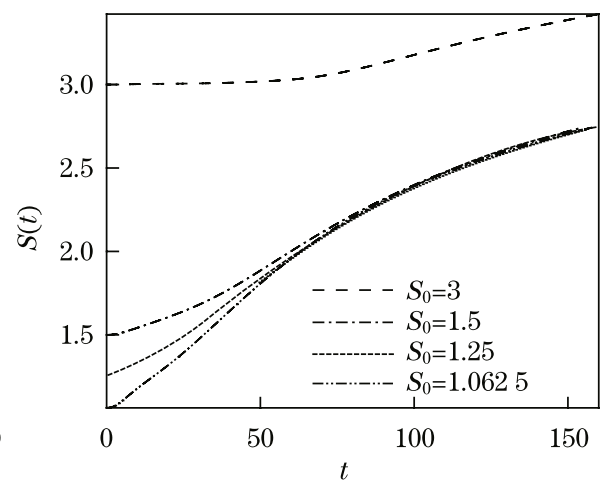

(b) $R e=1$ and $M a=100$

Fig. 11 Time evolutions of the separating distances between the drops

\subsection{Influence of thermal convection for $\Phi \neq 0$}

In this subsection, the full three-dimensional problem with $R e=1, M a=20$, and $S_{y 0}=$ $S_{z 0}=1.35$ is studied. In this simulation, both drops have zero velocities in the $x$-direction. In the vertical direction, the upper drop 1 migrates slower than the isolated drop, while the 
lower drop 2 moves faster than the isolated drop (see Fig. 12). As a result, the vertical distance between the two drops always decreases (see the analysis in the next subsection).

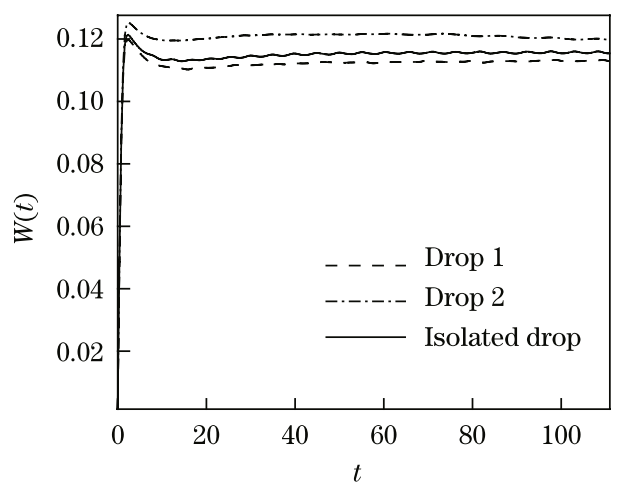

Fig. 12 Evolution of the migration velocity in the $z$-direction with $R e=1, M a=20$, and $S_{y 0}=$ $S_{z 0}=1.35$

The isotherms at $t=60$ are shown in Fig. 13. It can be seen that the thermal convection of the lower left drop 2 causes the bending of the isotherms around the upper right drop 1. Compared with that of the isolated drop, the temperature gradient on the lower part of the left drop 2 is reduced, while that on the upper part of the right drop 1 is enlarged. To get a better understanding of the velocities in the $z$-direction $(W)$, we study the temperature distributions at $t=60$ in the $x=0$ plane (see Fig. 14). Compared with that of the isolated drop, the temperature difference between the front and rear stagnation points of drop 2 is larger, while that of drop 1 is smaller.

The drop velocities in the $y$-direction $(V)$ are plotted in Fig. 15. It is found that drop 1 is always trying to move away from drop 2 in the $y$-direction. Drop 2 moves towards drop 1 in the beginning but starts to move away since $t \approx 30$. Because $V_{\mathrm{drop} 2}$ is always larger than $V_{\mathrm{drop} 1}$, the horizontal distance between the two drops increases throughout the simulation.

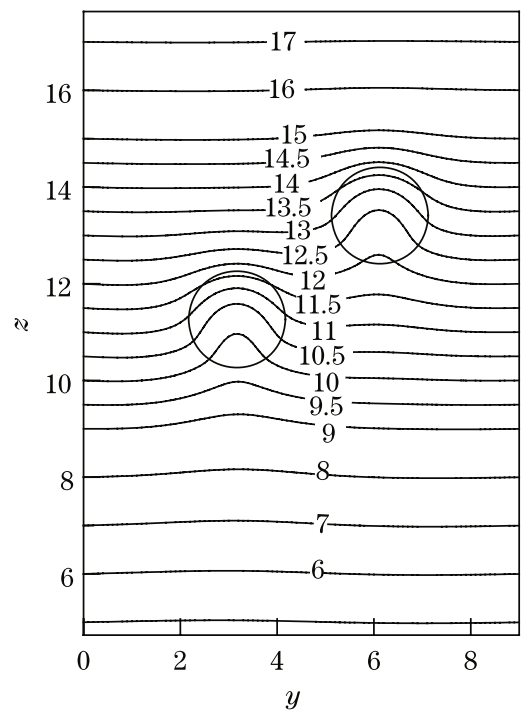

Fig. 13 Isotherms at $t=60$ with $R e=1, M a=20$, and $S_{y 0}=S_{z 0}=1.35$ 

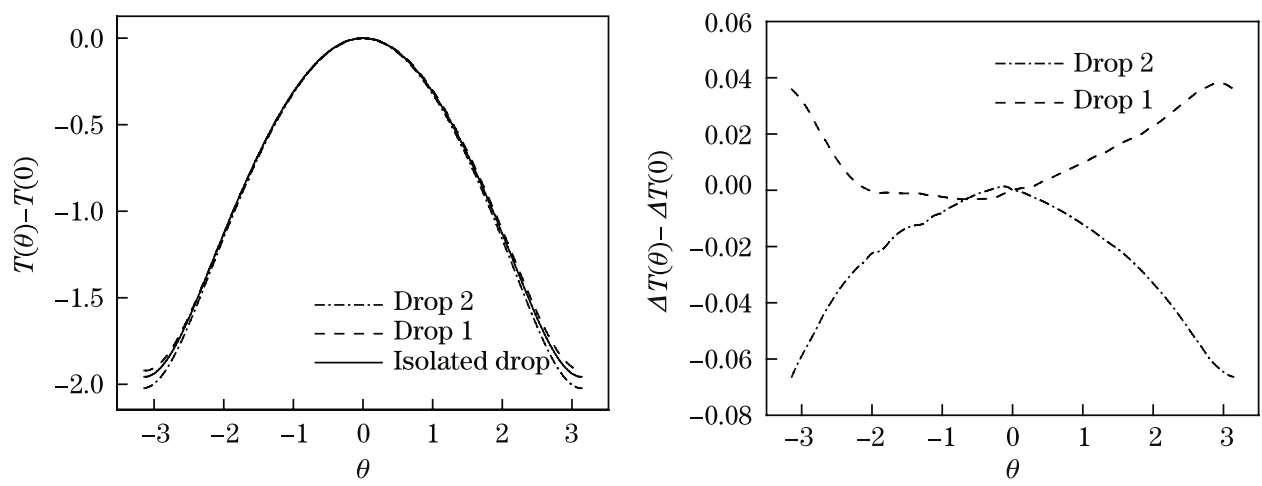

Fig. 14 Temperature distributing on the surface of the drops in the $x=0$ plane at $t=60$ with $R e=1, M a=20, S_{y 0}=S_{z 0}=1.35$, and $\Delta T(\theta)=T(\theta)-T_{\text {iso }}(\theta)$

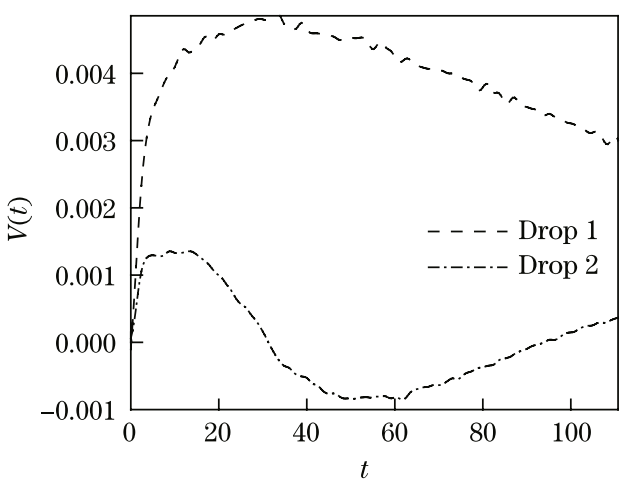

Fig. 15 Evolution of the migration velocity in the $y$-direction with $R e=1, M a=20$, and $S_{y 0}=$ $S_{z 0}=1.35$

\subsection{Influence of the initial distance for $\boldsymbol{\Phi} \neq \mathbf{0}$}

In this subsection, three sets of simulations with $R e=1$ and $M a=20$ starting from different initial distances are studied.

Case $1 \quad\left(S_{y 0}, S_{z 0}\right)=(1.35,1.35)$.

Case $2 \quad\left(S_{y 0}, S_{z 0}\right)=(1.1,1.35)$.

Case $3 \quad\left(S_{y 0}, S_{z 0}\right)=(1.35,1.25)$.

It is clear that the smaller the initial horizontal distance, the larger the velocities of the two drops in the $y$-direction, and the bigger the temperature differences $(T(\theta)-T(-\theta))$ between the left and right sides of the drops (see Fig. 16).

With different $R e$ and $M a$, the evolutions of the distances between the two drops for case 1 are shown in Fig. 17. It can be seen that the two drops separate very slowly when $R e=1$ and $M a=1$. When $R e$ is increased, the two drops get close fast in the vertical direction, while there are only trivial changes in the separated distances for the increasing $M a$.

Generally speaking, in the $z$-direction, the lower drop 2 moves faster than the isolated drop, while the upper drop 1 moves slower than the isolated drop. Thus, $S_{z}$ decreases throughout any simulation in this subsection. If the simulation domain is big enough, drop 2 would exceed drop 1 in the $z$-direction and slow down at a velocity smaller than that of drop 1 . Then, drop 1 will start to catch up with drop 2. Eventually, both drops will reach a steady migration state when they are aligned horizontally, as indicated in [10-11]. 


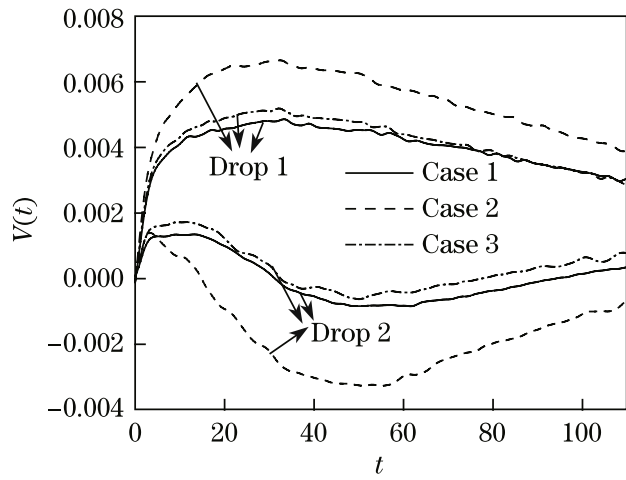

(a) Time evolutions

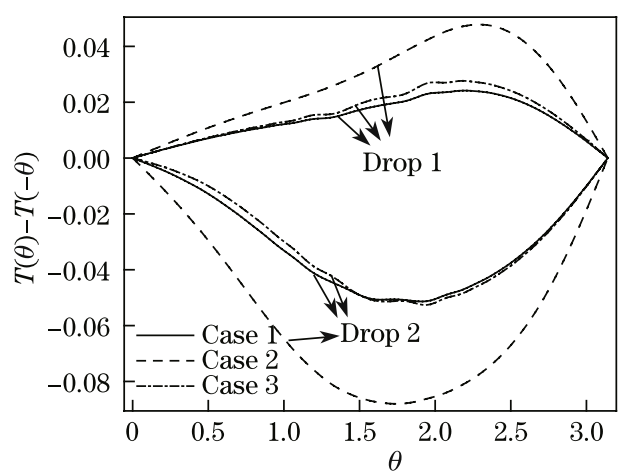

(b) Temperature differences

Fig. 16 Time evolutions of the vertical velocities of the drops with various initial distances and temperature differences between the left and right sides of the drops

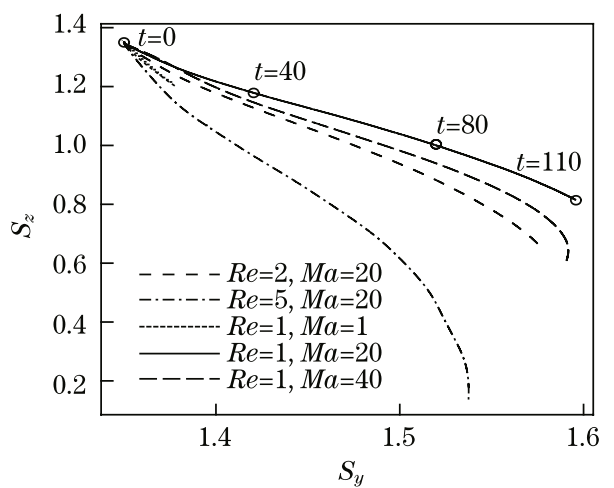

Fig. 17 Time evolution of the vertical and horizontal distances between the two drops with $S_{y 0}=$ $S_{z 0}=1.35$

\section{Conclusions}

In this paper, the interaction of two nondeformable drops in the thermocapillary motion is studied. When the inertia and thermal convections are neglected, the two vertically-placed drops move faster than the isolated drop, while the two horizontally-placed drops move slower. For the finite $M a$ and $\Phi=0$, the leading drop moves faster than the isolated drop, while the trailing drop migrates slower than the isolated drop due to the disturbed temperature field left by the leading drop. When the two drops are closer, their interaction is stronger. However, this intensive interaction will not last long, because the velocity difference of the two drops is also big. Once there is a sufficiently big gap between the two drops, they will migrate like the isolated drops. For the finite $M a$ and $\Phi \neq 0$, the motion of the two drops is still limited in the $y$ - $z$ plane. The upper drop 1 migrates slower while the lower drop 2 migrates faster than the isolated drop, which results in a smaller vertical distance and a bigger horizontal distance between the two drops. Table 1 sums up the velocities of drops 1 and 2 in the $z$-direction $\left(W_{1}, W_{2}\right)$ studied in this paper. In Table 1 , ' $^{\prime} /{ }^{\prime}-$ ' means that the velocity is bigger/smaller than $W_{\text {iso }}$. The velocities of rigid spheres in the Stokes flow are listed in the last row, and the bigger/smaller velocity stands for the resistance smaller/bigger than that on the isolated rigid sphere. 
Here, we only explore a few interacting mechanisms of two drops with a limited number of parameters. A wider range of parameters as well as the deeper physical explanations should be included in the future work.

Table 1 Velocities of drop $1\left(W_{1}\right)$ and drop $2\left(W_{2}\right)$ compared with $W_{\text {iso }}$

\begin{tabular}{cccccc}
\hline \multirow{2}{*}{ Parameter } & \multirow{2}{*}{$R e=M a=10^{-3}$} & \multicolumn{4}{c}{$R e=1$} \\
\cline { 3 - 5 } & & $M a=1$ & $M a=20$ & Sphere \\
\hline$\Phi=0$ & $W_{1}+, W_{2}+$ & $W_{1}+, W_{2}+$ & $W_{1}+, W_{2}-$ & $W_{1}+, W_{2}-$ & $W_{1}+, W_{2}+$ \\
$\Phi=0.68, \pi / 4,0.82$ & - & - & $W_{1}-, W_{2}+$ & - & $W_{1}+, W_{2}+$ \\
$\Phi=\pi / 2$ & $W_{1}-, W_{2}-$ & - & - & - & $W_{1}+, W_{2}+$ \\
\hline
\end{tabular}

\section{References}

[1] Young, N. O., Goldstein, J. S., and Block, M. J. The motion of bubbles in a vertical temperature gradient. J. Fluid Mech., 11, 350-356 (1959)

[2] Yin, Z. H., Gao, P., Hu, W. R., and Chang, L. Thermocapillary migration of nondeformable drops. Phys. Fluids, 20, 082101 (2008)

[3] Meyyappan, M., Wilcos, W. R., and Subramanian, R. S. The slow axisymmetric motion of two bubbles in a thermal gradient. J. Colloid Interface Sci., 94, 243-257 (1983)

[4] Meyyappan, M. and Subramanian, R. S. The thermocapillary motion of two bubbles oriented arbitrarily relative to a thermal gradient. J. Colloid Interface Sci., 97, 291-294 (1984)

[5] Balasubramaniam, R. and Subramanian, R. S. Axisymmetric thermal wake interaction of two bubbles in a uniform temperature gradient at large Reynolds and Marangoni numbers. Phys. Fluids, 11, 2856-2864 (1999)

[6] Anderson, J. L. Droplet interactions in thermocapillary motion. Int. J. Multiphase Flow, 11, 813-824 (1985)

[7] Keh, H. J. and Chen, S. H. The axisymmetric thermocapillary motion of two fluid drops. Int. J. Multiphase Flow, 16, 515-527 (1990)

[8] Keh, H. J. and Chen, S. H. Droplet interactions in axisymmetric thermocapillary motion. J. Colloid Interface Sci., 151, 1-16 (1992)

[9] Zhou, H. and Davis, R. H. Axisymmetric thermocapillary migration of two deformable viscous drops. J. Colloid Interface Sci., 181, 60-72 (1996)

[10] Nas, S. and Tryggvason, G. Thermocapillary interaction of two bubbles or drops. Int. J. Multiphase Flow, 29, 1117-1135 (2003)

[11] Nas, S., Muradoglu, M., and Tryggvason, G. Pattern formation of drops in thermocapillary migration. Int. J. Heat Mass Transfer, 49, 2265-2276 (2006)

[12] Balasubramaniam, B., Lacy, C. E., Woniak, G., and Subramanian, R. S. Thermocapillary migration of bubbles and drops at moderate values of the Marangoni number in reduced gravity. Phys. Fluids, 8, 872-880 (1996)

[13] Brady, P. T., Herrmann, M., and Lopez, J. M. Confined thermocapillary motion of a threedimensional deformable drop. Phys. Fluids, 23, 022101 (2011)

[14] Gao, P. Numerical Investigation of the Drop Thermocapillary Migration (in Chinese), Ph. D. dissertation, Chinese Academy of Sciences (2007)

[15] Hick, W. M. On the motion of two spheres in a fluid. Phil. Trans. Roy. Soc., 171, 455-492 (1880)

[16] Herman, R. A. On the motion of two spheres in fluid and allied problems. Quart. J. Pure Appl. Math., 22, 204-262 (1887)

[17] Kaneda, Y. and Ishii, K. The hydrodynamic interaction of two spheres moving in an unbounded fluid at small but finite Reynolds number. J. Fluid Mech., 124, 209-217 (1982)

[18] Batchelor, G. K. An Introduction to Fluid Mechanics, Cambridge University Press, Cambridge, (1967) 
[19] Wu, W. Y. Fluid Dynamics (in Chinese), Peking University Press, Beijing (1983)

[20] Happle, J. and Brenner, H. Low Reynolds Number Hydrodynamics, 1st ed., Martinus Nijhoff Publishers, The Hague (1965)

[21] Yan, Z. Y. Theory of Low Reynolds Number Hydrodynamics (in Chinese), Peking University Press, Beijing (2002)

[22] Stimson, M. and Jeffery, G. B. The motion of two spheres in a viscous fluid. Proc. Roy. Soc., Lond. A, A111, 110-116 (1926)

[23] Goldman, A. J., Cox, R. G., and Brenner, H. The slow motion of two identical arbitrarily orientedspheres through a viscous fluid. Chem. Eng. Sci., 21, 1151-1170 (1966) 